\title{
Mycobacterium tuberculosis and pemphigus vulgaris
}

\author{
Katarzyna Osipowicz, Cezary Kowalewski, Katarzyna Woźniak \\ Department of Dermatology and Immunodermatology, Medical University of Warsaw, Warsaw, Poland \\ Adv Dermatol Allergol 2018; XXXV (5): 532-534 \\ DOI: https://doi.org/10.5114/ada.2018.72744
}

Tuberculosis (TB) is a disease caused by Mycobacterium tuberculosis (M. tuberculosis) affecting principally the lungs. Recently, it has been postulated that $M$. tuberculosis causes more deaths than any other infectious disease [1]. Patients with smear-positive sputum for $M$. tuberculosis are the main source of infection. Normally, the therapy takes 6 to 9 months using isoniazid (INH), rifampicin (RMP), ethambutol (EMB), and pyrazinamide (PZA). For the drug-resistant forms of TB, treatment consists of a combination of fluoroquinolones with other injectable medications, such as kanamycin, capreomycin or amikacin [2].

Pemphigus vulgaris (PV) belongs to a group of acantholytic bullous dermatoses, with a potentially fatal outcome. The disease is characterized by flaccid bullae formation observed within the epidermis, being a result of IgG autoantibody production directed against desmoglein 1 and 3, expressed in the epidermis and, particularly, the mucosal epithelia.

In most patients, PV develops spontaneously [3]. Inducing or triggering factors, i.e. viral infections, physical agents, contact allergens, stress, dietary factors, and drug intake have also been reported. The PV may be induced by three groups of drugs, containing a sulfhydryl group, a phenol group and, finally, a non-phenol group [3].

We present the case of a patient with PV provoked by rifampicin taken due to pulmonary TB.

A 48-year-old male with a 2-year history of pulmonary TB treated with INH and RMP, and 1-year history of an active $P V$ persistently treated with prednisone at a dose of $80 \mathrm{mg}(1 \mathrm{mg} / \mathrm{kg})$ and azathioprine at a dose of $100 \mathrm{mg}$. Despite that therapy, he still presented erosions located in the oral mucosa and on the trunk and extremities (Figure $1 \mathrm{~A}-\mathrm{C}$ ). The activity of PV was confirmed by direct and indirect immunofluorescence (Figure 2) study showing in vivo bound and circulating intercellular IgG antibodies at a titer of 1280 . The increase in the dose of azathioprine to $150 \mathrm{mg} /$ day did not lead to the improvement. After pulmonary consultation, antituberculotic medicines; were discontinued. One month later, we observed a significant improvement of the patient and a decreased level of pemphigus antibodies. Currently, the patient is in clinical remission of PV (Figures $1 \mathrm{D}-\mathrm{F}$ ) and takes $30 \mathrm{mg} /$ day of prednisone and $100 \mathrm{mg} /$ day of azathioprine. The remission of TB has been confirmed by chest radiography.

Mycobacterium tuberculosis infection is one of the most common infections in the world and is one of top 10 causes of death worldwide. Only in 2014, over 9.6 million people developed TB and 1.5 million died [2]. The recent guidelines recommend two-stage treatment for patients with pulmonary TB: the first phase of intensive treatment - minimum 2 months with 4 medications: RMP, INH, PZA and EMB as a sterilizing treatment. The second phase of treatment is a minimum of 4-month therapy with rifampicin and isoniazid. In total, it should not be shorter than 6 months [2]. Our patient has been treated with INH and RMP for 1 year. All the anti-TB drugs may be responsible for numerous side effects; however, they are rather mild. INH may cause skin rash or toxic fever, PZA - rash, urticariat and pruritus [4]. In turn, EMB may provoke dermatitis, erythema multiforme, pruritus as well as severe hematological diseases such as hemolytic anemia, and also can cause slight hyperuricemia and, uncommonly, dose-related retrobulbar neuritis? [5].

In the case of RMP, along with itching and flushing, much more severe disorders, such as hepatotoxicity, pseudomembranous colitis, and porphyria were also reported [6] since RMP is being metabolized in the liver and can penetrate tubercular foci, lymph nodes and reaches body fluids [3].

Dermatologic side effects of RMP, including PV, results from hypersensitivity. However, it is not sufficient to initiate the autoimmune process, which was reported in the case of pemphigus in only one of the twins, or in two of three siblings with identical haplotypes prone to PV [3]. It is well documented that for the initiation of $P V$ in genetically predisposed people an external agent is required, mainly a drug containing thiol groups, which directly affects desmogleins leading to blister formation and production of pemphigus antibodies [7]. That mech-

Address for correspondence: Katarzyna Osipowicz MD, Department of Dermatology and Immunodermatology, Medical University of Warsaw, 82 a Koszykowa St, 02-008 Warsaw, Poland, phone: +48 666092 732, e-mail: osipowicz.kasia@gmail.com Received: 24.06.2017, accepted: 12.07.2017. 

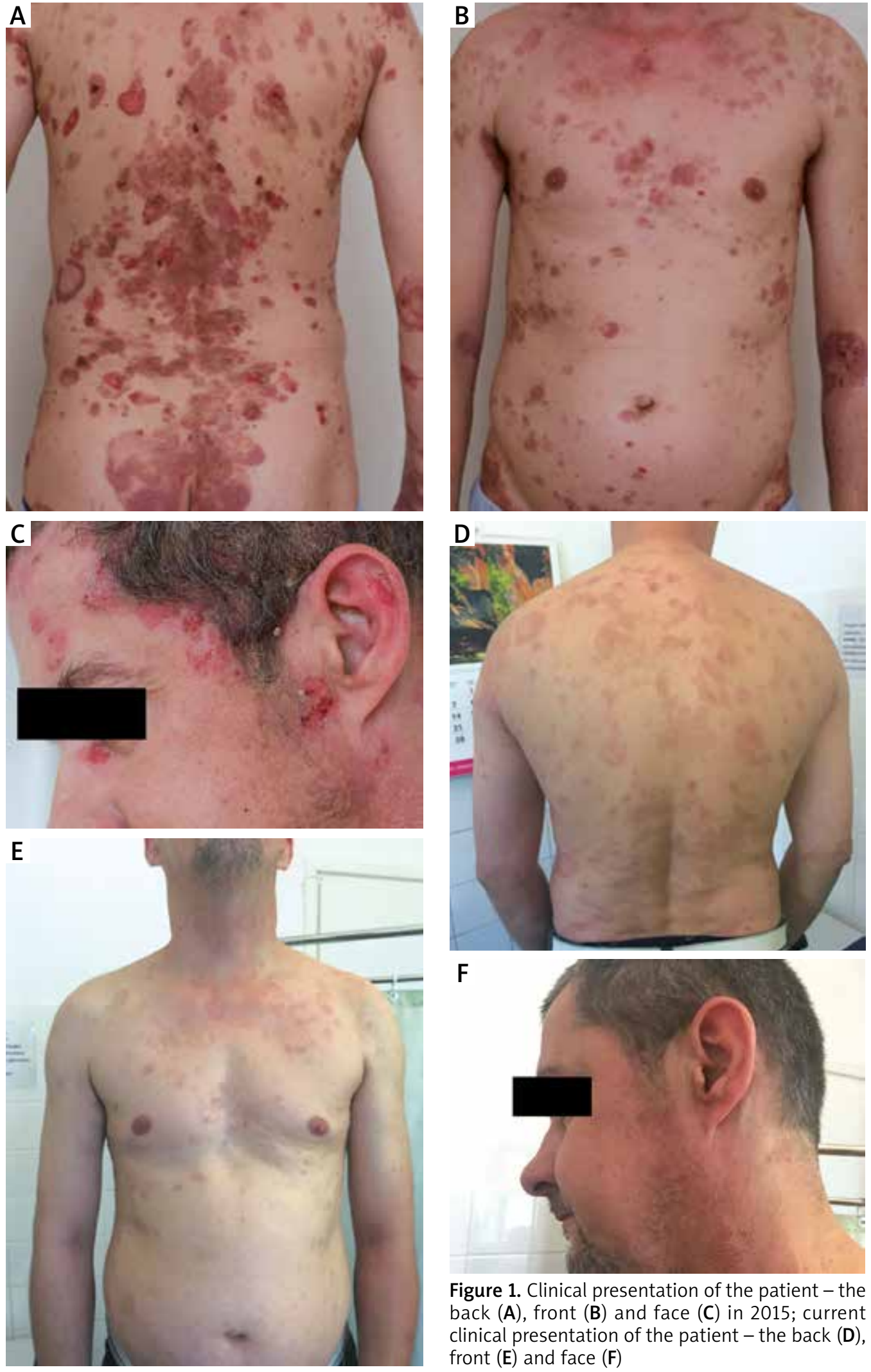

Figure 1. Clinical presentation of the patient - the back (A), front (B) and face (C) in 2015; current clinical presentation of the patient - the back (D), front $(\mathrm{E})$ and face $(\mathrm{F})$ 


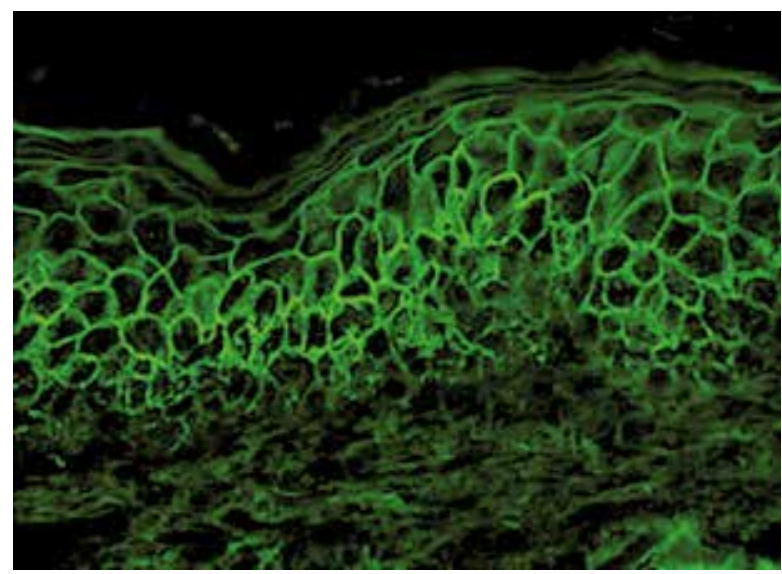

Figure 2. In vivo bound IgG located in intercellular spaces of epidermis characteristic for pemphigus vulgaris

anism is also postulated in the case of dietary factors playing a role in PV provocation, particularly hot spices, rich in thiols and isothiocyanates groups. It was also documented that physical agents, such as contact allergens, UV or ION radiation, thermal or electrical burns and even beauty treatments may provoke PV [3].

On the other hand, infections, especially herpes or bronchiolitis can also cause the appearance of a new outbreak or exacerbation of pemphigus [3]. Viruses and bacteria can stimulate the immune response through cytokine production, which leads to induction of human leucocyte antigen type 2 expression in keratinocyte membranes. Additionally, viral infections can directly infect $B$ and $T$ lymphocytes, leading to the production of autoreactive $B$ lymphocytes and pemphigus antibody production. On the other hand, patients suffering from $\mathrm{PV}$ are more prone to $M$. tuberculosis infection.

Our patient suffering from TB and treated with INH and RMP developed PV 1 year later. The diagnosis of PV was established based on clinical features, and direct and indirect immunofluorescence as per recently published guidelines [8]. Despite the proper therapeutic regimen for PV, no clinical improvement was observed during 12 months. It is highly likely that long-lasting severe PV was caused by RMP, which belongs to a group of medicines known to be responsible for PV provocation [8]. Rifampicin administration results in abnormal liver function tests - elevated transaminases and alkaline phosphatase [9]. Therefore, we can consider that rifampicin diminishes the pharmacological effect of systemic glucocorticosteroids in our patient [10]. Rifampicin can also cause the elevation of serum pemphigus antibodies while decreasing glucocorticoid serum levels [10], therefore we did not observe either clinical improvement or side effects of glucocorticosteroids in our patient. Eventually, the withdrawal of rifampicin led to the clinical remission and negativization of pemphigus antibodies in the patient's serum. Currently, both PV and TBC are in remission.

In conclusion, it is necessary to be aware of numerous environmental factors including drugs and infections that may provoke PV, or drug interactions which may delay clinical and immunological remission.

\section{Conflict of interest}

The authors declare no conflict of interest.

\section{References}

1. McLean KJ, Munro AW. Drug targeting of heme proteins in Mycobacterium tuberculosis. Drug Discov Today 2017; 22: 566-75.

2. WHO. Global Tuberculosis Report 2016. World Health Organization, Geneva 2016.

3. Ruocco V, Ruocco E, Lo Schiavo A, et al. Pemphigus: etiology, pathogenesis, and inducing or triggering factors: facts and controversies. Clin Dermatol 2013; 31: 374-81.

4. Mack R, Holdiness MD. Adverse cutaneous reactions to antituberculosis drugs. Int J Dermatol 1985; 24: 280-5.

5. Sivakumaran P, Harrison AC, Marschner J, et al. Ocular toxicity from ethambutol: a review of four cases and recommended precautions. NZ Med J 1998; 111: 428-30.

6. O'Brien RJ, Long MW, Cross FS, et al. Hepatotoxicity from isoniazid and rifampin among children treated for tuberculosis. Pediatrics 1983; 72: 491-9.

7. Brenner S, Sasson A, Sharon O. Pemphigus and infections. Clin Dermatol 2002; 20: 114-8.

8. Kowalewski C, Dmochowski M, Placek W, et al. Diagnosis and therapy of pemphigus - consensus of Polish Dermatological Society. Przegl Dermatol 2014; 101: 147-55.

9. Gange RW, Rhodes EL, Edwards CO, et al. Pemphigus induced by rifampicin. Br J Dermatol 1976; 95: 445-8.

10. Miyagawa S, Yamashina Y, Okuchi T, et al. Exacerbation of pemphigus by rifampicin. Br J Dermatol 1986; 114: 729-32. 